\title{
The Effect of Doped by Nano and Micro Zno Particles on Optical and Structural Properties of PVA Films
}

\author{
Ilham K. Onees ${ }^{1}$ \\ ${ }^{1}$ Department of Electronics \& Communication, Erbil Technology College, Erbil Polytechnic \\ University, Erbil, Iraq \\ Correspondence: Ilham K. Onees, Erbil Polytechnic University, Erbil, Iraq. \\ Email: ilham.onees@epu.edu.iq
}

Doi: 10.23918/eajse.v6i1p153

\begin{abstract}
This paper studied the effects of nano and micro $\mathrm{ZnO}$ particles dopant on optical properties and structure of (PVA) films that were prepared for both pure and $\mathrm{ZnO}$ doped PVA using solvent casting method. The scanning electron microscopy is employed to characterize the morphology properties for the prepared samples. X-Ray diffraction technique was used to study the structure of films and the effect of ZnO doped on it. X-ray diffraction analysis confirmed that these films are polycrystalline structure nature. Optical energy gap PVA/ZnO films were calculated from absorptions spectra. It was found that the films exhibit indirect allowed transitions gap which decreased with $\mathrm{ZnO}$ particles doped.
\end{abstract}

Keywords: PVA, Nano and Micro Zno Particles, Casting Method

\section{Introduction}

To create a new material, there has been a great interest of material research due to the characteristics connected to a specific application to understand both physical and chemical techniques which specifies its characteristics. Due to its distinctive uses for industrial and technological applications, Polymers has paved a way for extensive field of research, because Polyvinyl alcohol (PVA) is the most frequently polymeric material used poly (methyl methacrylate) (MMA) and polyvinyl chloride (PVC) (Meyer, 2009). Mute et al. (2004) noted that, in scientific and technological researches, polymer materials are of great interest, optical communications in clouding polymer optical fiber, optical waveguides and their ease of processing (Raja, Sarma, \& Narasimh, 2003). The PAV is semi crystalline, synthetic, water soluble polymer and it has a very important application (Makled \& ElMans, 2013). Modifying the organic polymers consider the easier and alternatives technologies by including the metal or metal chelates or by incorporating the metal into side groups. Generally, to improve the behavior of polymer and as a norm of composition materials, whether atomic or ionic state, a metal could be introduced into a polymer matrix, which improves new properties with both matrix and filler ones (Muna et al., 2014). ZnO crystallizes can be found in the hexagonal wurtzite lattice that is used in the thin film industry as a transparent for conducting oxide or as a catalyst in methanol synthesis, or in the rock salt structure (Kim, Park, \& Ma, 1997). When considering the properties of nanosized $\mathrm{ZnO}$ particles, the nature of the surface is essential. These are results of high surface area to volume ratio of $\mathrm{ZnO}$ nano-structures. The reports show that there are several methods for synthesizing $\mathrm{ZnO}$ nano-sized. The $\mathrm{ZnO}$ nano-sized demonstrate anovative properties and potential applications. Several methods like the chemical vapour transport and condensation process templatebased growth method, and wet chemical have synthesized the $\mathrm{ZnO}$ nano-sized (Jackson, 2006). Hydrogen bonding was used between the $\mathrm{OH}$ groups of PVA and the functional groups of metal oxides to prepare the Polymer/ZnO composites (Chandrakala et al., 2013). However, changing polymer ratio was used for modifying the optical and electrical characteristics of polymer composites (Mansour, 
Mansour, \& Abdo, 2015; Gong et al., 2014). The energy band gap of polymers/ZnO composites is red shifted and decreases as the particle size of $\mathrm{ZnO}$ increases in the polymer composites (Shanshool et al., 2017). The main aim for this study are to control the values of indirect energy gap of PVA by using different particle size of $\mathrm{ZnO}$ doped PVA.

In the present work, we prepared PVA/ZnO films by easy and inexpensive method and study the effect of nano and micro $\mathrm{ZnO}$ particles dopant on the impact of morphology as well as in the structure, and optical properties of $\mathrm{PVA} / \mathrm{ZnO}$ films.

\section{Experimental}

Pure and doped films were formed by using casting method. To dope materials, PVA with molecular weight of $107 \mathrm{~g} / \mathrm{mol}$ from $(\mathrm{BDH})$ company, as a host polymeric; micro $(60 \mu \mathrm{m})$ with nano $(50 \mathrm{~nm})$ $\mathrm{ZnO}$ particles were used. To obtain PVA solution, $0.2 \mathrm{~g}$ weight was suspended by using $5 \mathrm{ml}$ of purified water. The mixture was then stirrer for approximately $30 \mathrm{~min}$ at $80^{\circ} \mathrm{C}$. This was done to get homogenous solution. This was then transferred into a clean glass Petri dish with $6 \mathrm{~cm}$ diameter and dried for 3 hour at $70^{\circ} \mathrm{C}$. Doped films with nano and micro $\mathrm{ZnO}$ partials were fabricated by tow state, first by dissolving both PVA and $\mathrm{ZnO}$ partials in $5 \mathrm{ml}$ of distilled water at $80^{\circ} \mathrm{C}$ with $\mathrm{ZnO}$ ratio (4\%), second state by dissolving PVA in $4 \mathrm{ml}$ of distilled water at $80^{\circ} \mathrm{C}$ and dissolving $\mathrm{ZnO}$ in $1 \mathrm{ml}$ of methanol, then mixed the tow solution together. All solutions were transferred into a clean glass Petri dish and dried for 3 hours at $70^{\circ} \mathrm{C}$. Then, tweezers clamp were used to remove the dried homogeneous films were then removed easily by using, see Table (1).

Table 1: The PVA/ZnO films preparation conditions

\begin{tabular}{|c|c|c|c|c|}
\hline $\begin{array}{l}\text { Samples } \\
\text { Abbreviate } \\
\text { d }\end{array}$ & $\begin{array}{l}\text { Thickness } \\
(\mathrm{mm})\end{array}$ & $\begin{array}{l}\text { PVA } \\
\text { Weight (g) }\end{array}$ & $\begin{array}{l}\mathrm{ZnO} \\
\text { Weight (g) }\end{array}$ & Dissolving \\
\hline $\mathrm{A}$ & 0.049 & $0.2 \mathrm{gm}$ & 0 & \multirow{3}{*}{$5 \mathrm{ml}$ distal water } \\
\hline B & $0.051 \mathrm{Mic}$ & \multirow{2}{*}{0.192} & \multirow{2}{*}{0.008} & \\
\hline $\mathrm{C}$ & 0.052 nano & & & \\
\hline $\mathrm{D}$ & $0.056 \mathrm{Mic}$ & \multirow{2}{*}{0.192} & \multirow{2}{*}{0.008} & \multirow{2}{*}{$\begin{array}{l}4 \mathrm{ml} \text { distal water for PVA } \\
\text { and } 1 \mathrm{~m} \text { methanol for } \mathrm{ZnC}\end{array}$} \\
\hline $\mathrm{E}$ & 0.059 nano & & & \\
\hline
\end{tabular}

By using a digital micrometer, medium thickness of the prepared films was approximately $(0.0534$ $\mathrm{mm}$ ). A double beam UV/VIS spectrometer (Shimadzu, Japan) in the wavelength range (200-1000) $\mathrm{nm}$ for the absorptance and transmittance measurements. Philips X-ray diffractometer was used to measure the structural nature for the films. Scanning electron microscopy SEM was used to show the morphology of produced samples.

\section{Results and Discussion}

All samples images are shown in Fig. (1). a flat and clear surface morphology of the pure PVA film is obtained with a uniform distribution is observed as shown in Fig. (1-A). 


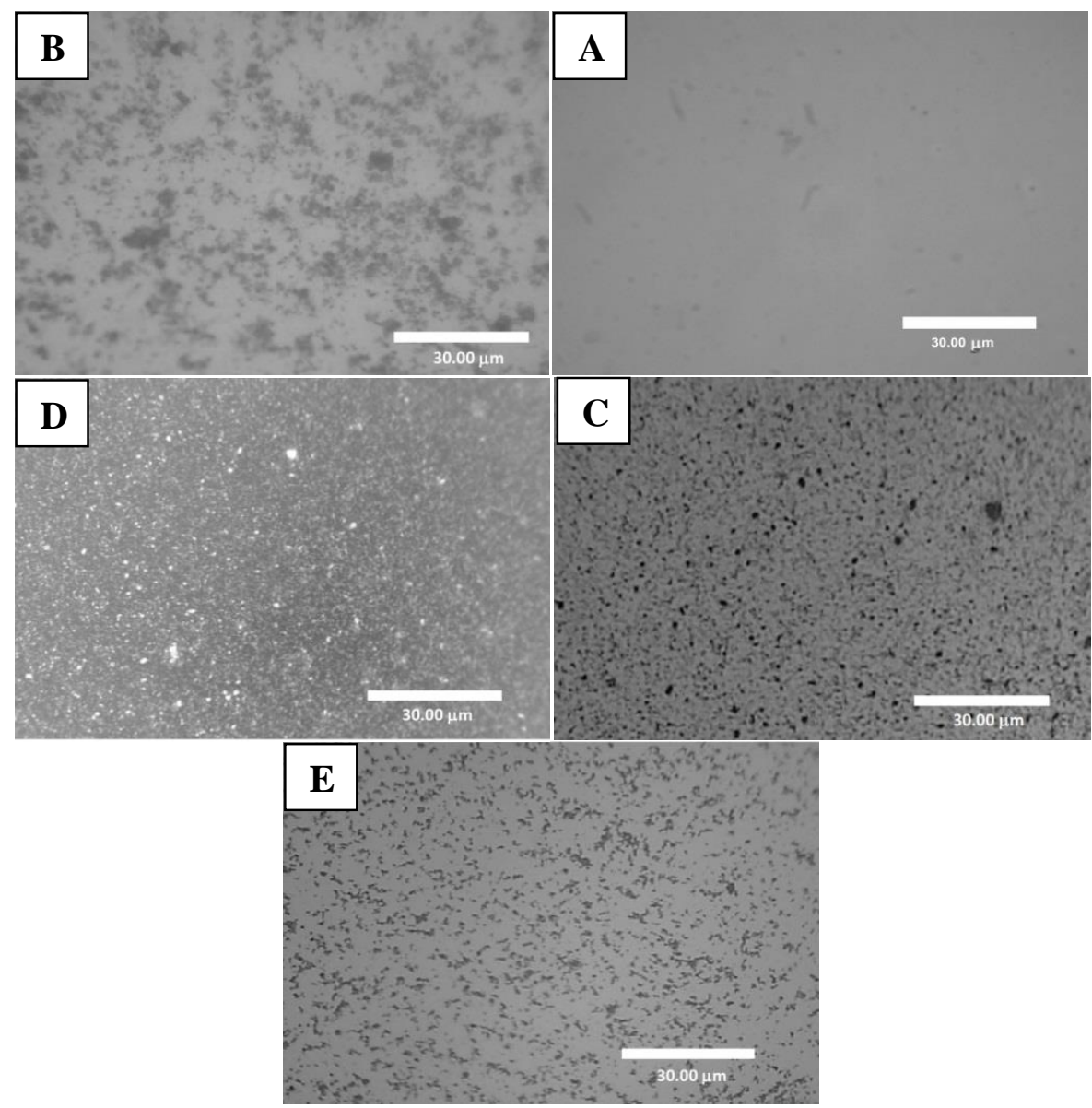

Figure 1: SEM image of PVA and PVA/ZnO films

The PVA/ZnO films' images (Figure 1-B, C) show that they were prepared by water solvent for both micro and nano $\mathrm{ZnO}$ particles. The conglomerate of $\mathrm{ZnO}$ particles are in nano state because of the van der waals force (Hong \& Kim, 2014), while these agglomerations of $\mathrm{ZnO}$ particles are significantly less than PVA/ZnO films that were prepared by water and methanol solvent for both micro and nano $\mathrm{ZnO}$ particles as in images $\mathrm{D}$ and $\mathrm{E}$. This means the water and methanol solvent is best for preparation of PVA/ZnO films.

The XRD pattern of PVA and PVA/ZnO films have been shown in Figure (2). Which show that, the peaks of the XRD were between $10^{\circ}$ and $80^{\circ}$. 


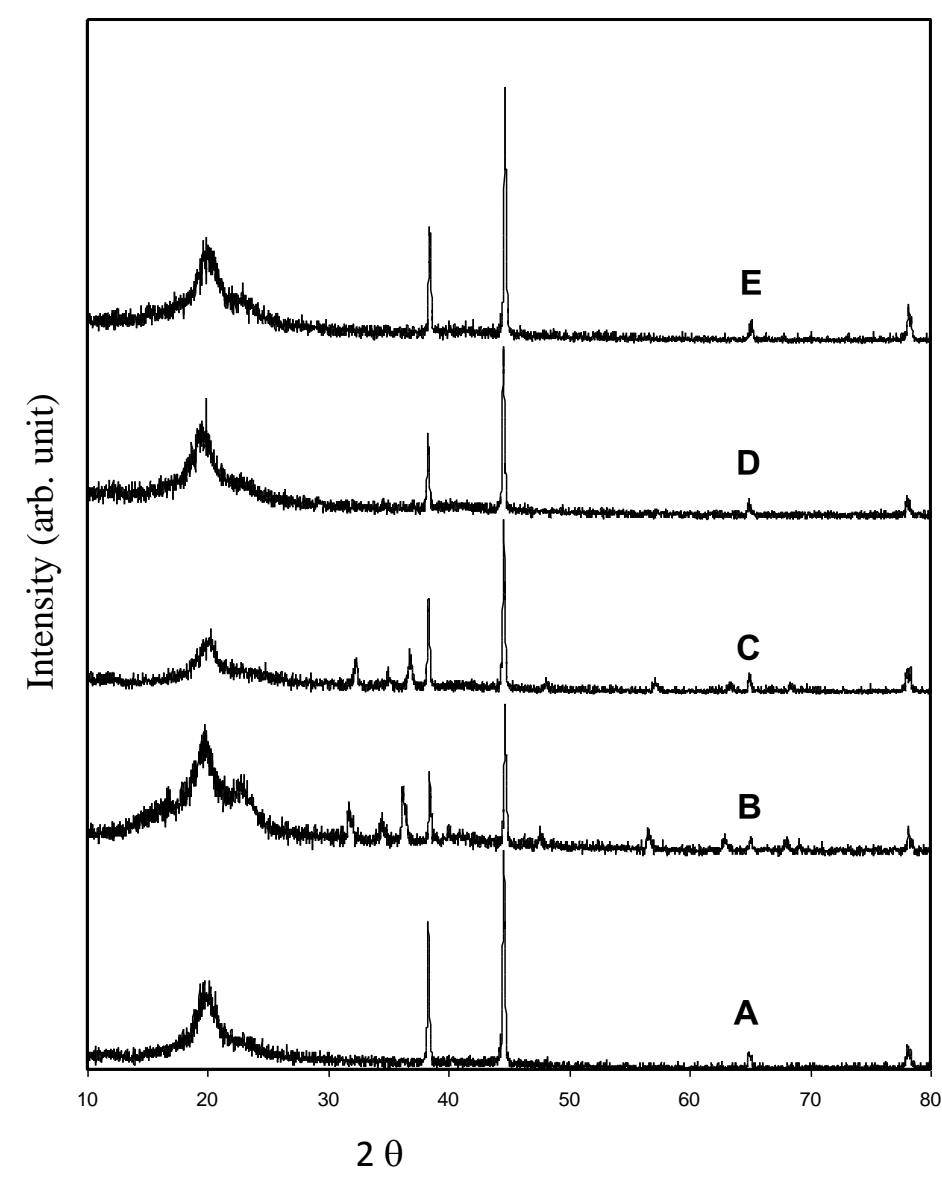

Figure 2: The XRD spectra of PVA and PVA/ZnO films

The diffraction peak indicates that, all films are polycrystalline structure with three main peaks, broad one in $2 \theta=19.6^{\circ}$ and two sharp peaks corresponding to the positions $2 \theta=38.2^{\circ}$ and $44.5^{\circ}$, it is clear that no amorphous phase is detected. When comparing between doped by micro and nano $\mathrm{ZnO}$ particles as shown in $(\mathrm{B}, \mathrm{C})$ and $(\mathrm{D}, \mathrm{E})$ patterns, it is noted that the intensity and sharpness of peaks increases, which means increase in crystallite size and this is confirmed when the crystallite size by using Scherrer equation is calculated (Cullity \& Stock, 2001) (see Table 2):

$$
\text { C.S. }=\frac{D \lambda}{\Delta \theta \cos \theta}
$$

The highest dissolution appears where angle of diffraction is $\theta, \Delta \theta$ is full-width at maximum half of the XRD, D Scherrer's constant, usually assume $\mathrm{D}=1$, and $\lambda$ the wave length of $\mathrm{X}$-ray.

Whereas when we compare between the spectra $(\mathrm{B}, \mathrm{D})$ and $(\mathrm{C}, \mathrm{E})$ where the dissolution method differs, we notice a decrease in the number of peaks when using methanol in preparing the sample, this means decrease in the polycrystalline structure state when using methanol in preparing. 
Table 2: The parameters of PVA and PVA/ZnO films

\begin{tabular}{|l|l|l|}
\hline $\begin{array}{l}\text { Samples } \\
\text { Abbreviated }\end{array}$ & Crystallite size $\mathrm{nm}$ & $\mathrm{Eg} \mathrm{eV}$ \\
\hline A & 52.58 & 4.8 \\
\hline B & 54.350 & 4.4 \\
\hline C & 59.23 & 3.7 \\
\hline D & 53.82 & 3.8 \\
\hline E & 60.012 & 3.4 \\
\hline
\end{tabular}

Figure (3), shows the optical absorbance (A) and transmission (T\%) of pure PVA and ZnO doped PVA films. From this figure it is clear that the absorbance of the films increasing with doped, and the effect of nano $\mathrm{ZnO}$ particles more than micro $\mathrm{ZnO}$ particles for both state of dissolving. In the same figure the optical transmittance spectra of PVA and PVA/ZnO films can be seen which show the opposite behavior in absorption spectra. The optical absorption coefficient $(\alpha)$ of films give more details for the electronic band structure, the band tail and energy gap, so it is very important. The absorption coefficient $(\alpha)$ can be determined like a function of frequency as in the formula (Susilawati \& Aris, 2009).

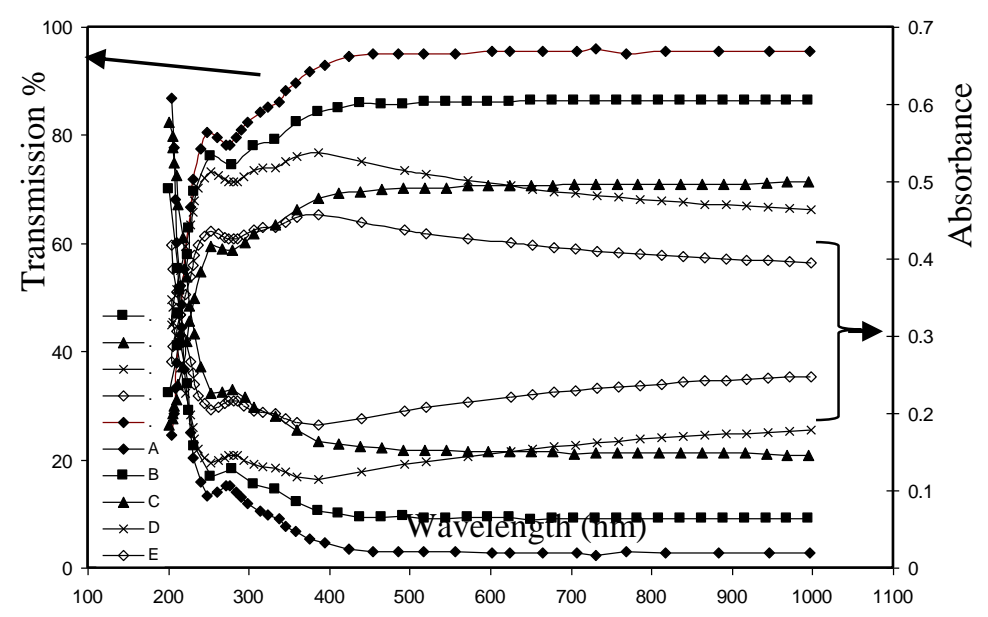

Figure 3: The FTIR Absorbance and Transmission (\%) spectra of PVA and PVA/ZnO

$$
\alpha=\frac{2.303 \mathrm{~A}}{\mathrm{~d}}
$$

Note: $\mathrm{d}$ is the film thickness while $\mathrm{A}$ is absorbance.

The absorption coefficient relates to the nature of electronic transitions. The values of the absorption coefficient show us the type of the electron transitions, if the values of $(\alpha)$ greater than $10^{4} \mathrm{~cm}^{-1}$ which means that, for $(\alpha)$ less $10^{4} \mathrm{~cm}^{-1}$ the indirect transitions occur and direct transitions takes place in materials (Tauc, 1979; Sze, 2007). For both crystalline and amorphous materials the energy gap $E_{\mathrm{g}}$ for direct and indirect transitions could be detected via the optical absorption spectra.

Figure (4) shows the absorption coefficient $(\alpha)$ as a function of photon energy. It was seen that $(\alpha)$ is increased because of $\mathrm{ZnO}$ doped. The results show the value of $(\alpha)$ for pure and doped PVA films is 
less than $\left(10^{4} \mathrm{~cm}^{1}\right)$. To prove the nature of optical transition; the optical data was analyzed by using the relation (Tauc, 1979; Sze, 2007):

$$
\alpha \square v=B\left(\square v-E_{g}\right)^{m}
$$

Where: $\mathrm{h}=$ planck constant, $\mathrm{Eg}=$ optical energy band gap. $\mathrm{B}=$ disorder parameter (constant)

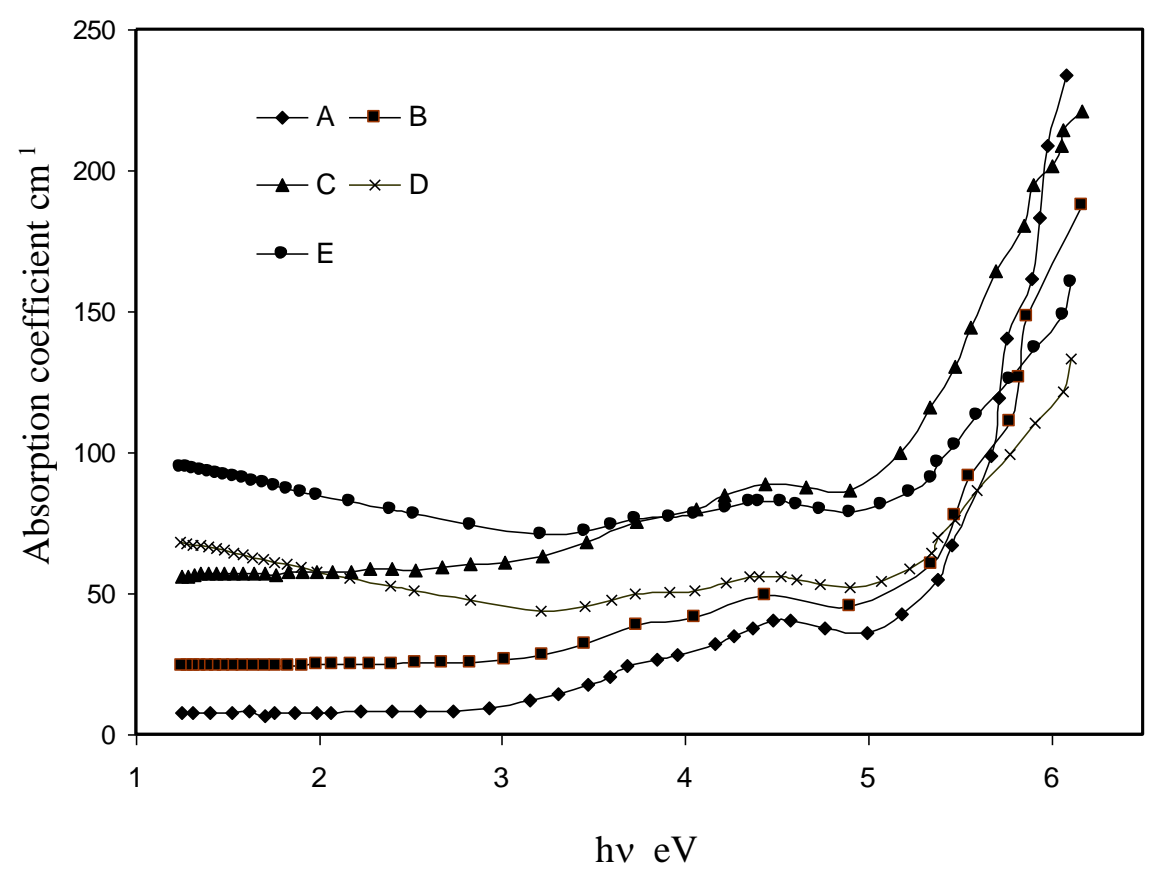

Figure 4: The Absorption coefficient spectra of PVA and PVA/ZnO films

The power coefficient which can be defined via kind of possible electronic transitions, i.e., $m=1 / 2$, $3 / 2$, 2 or $1 / 3$ for allowed and forbidden transitions for both direct and indirect (Kim, Park, \& Da, 1997). The variation of $(\alpha h v)^{1 / 2}$ for PVA and PVA/ZnO films as a function of photon energy is shown in Figure (5). Extrapolation of the linear portion of this curve to a point $(\alpha h v)^{1 / 2}=0$, shows the optical energy band gap $\left(E_{g}\right)$ for the films. All the change in optical parameters of the films can attribute to the effect of $\mathrm{ZnO}$ doped on the structure of PVA. 


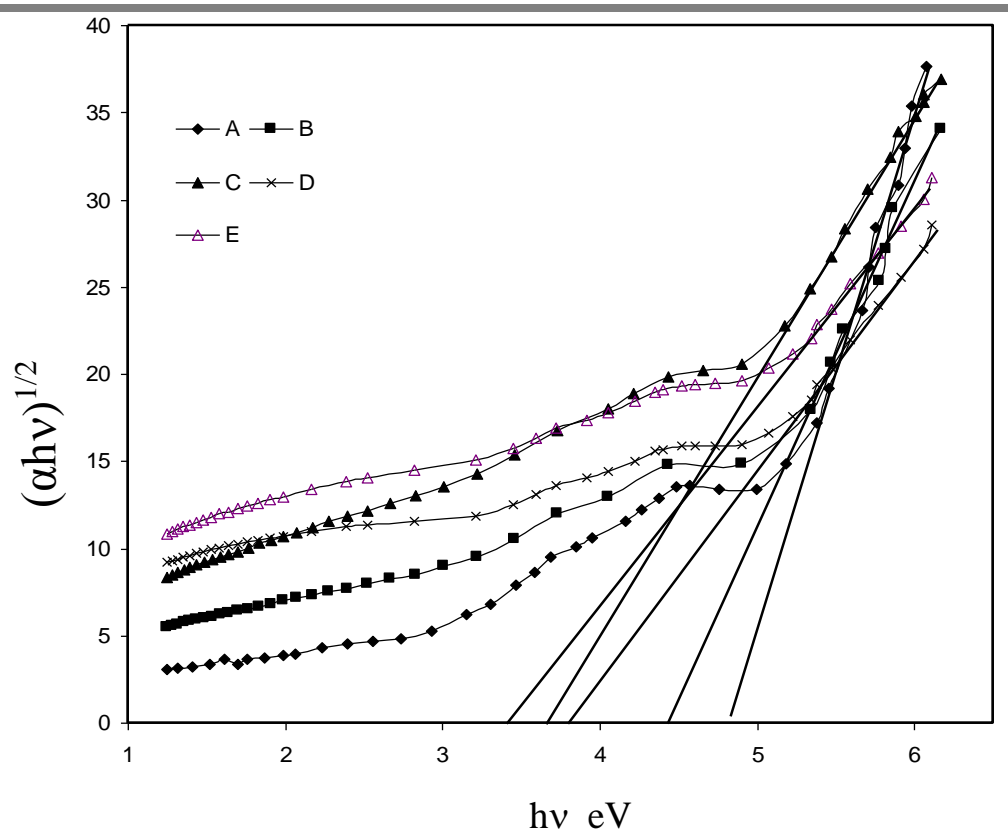

Figure 5: The $(\alpha h v)^{1 / 2}$ vs. hv of PVA and PVA/ZnO films

\section{Conclusion}

The PVA and PVA/ZnO films could be successfully composed via molding procedure under effect of doped by nano and micro $\mathrm{ZnO}$ particles and dissolving method; the systematic study was conducted for morphology, structure, and optical properties for prepared samples. The XRD show polycrystalline structure samples. From optical properties found, the doping process decreases the transmission, while the absorbance increases with doping. It has been found that the type of electronic transition is an indirect allowed transition and the power space is reduced with doping. On the other hand, the effect of doping with nano $\mathrm{ZnO}$ particles was more effective on the physical properties of PVA from micro $\mathrm{ZnO}$ particles; in addition to that, solubility using methanol in preparing samples is better than using water alone.

\section{References}

Chandrakala, H., Ramaraj, B., Shivakumaraiah, J., \& Siddaramaiah, L. (2013). Polyvinyl alcohol/carbon coated zinc oxide nanocomposites: electrical, optical, structural and morphological characteristics. J. Alloys Compd. 580, 392-400.

Cullity, B., \& Stock, S. (2001). Elements of X-ray Diffraction. 3rd ed. USA: Prentice-Hall.

Gong, X., Tang, C., Pan, L., Hao, Z., \& Tsui, C. (2014). Characterization of poly (vinylalcohol) (PVA)/ZnO nanocomposites prepared by a one-pot method. Composites Part B, 60, 144149.

Hong, Y., \& Kim, K. (2014). Architectured van der Waals epitaxy of ZnO nanostructures on hexagonal BN. NPG Asia Mater 6, e145.

Jackson, M. (2006). Microfabrication and nanomanufacturing. Taylor \& Francis Group, LLC.

Kim, K., Park, K., \& Ma, D. (1997). Structural, Electrical and Optical properties of Aluminum doped Znic Oxid films by radio frequency magnetron sputtering. J. Appl.Phys. 81(12), 7764.

Makled M. H., \& El-Mansy, M.K. (2013). Electrical conduction and dielectric relaxation in p-type PVA/CuI polymer composite. Journal of Advanced Research, 4, 531-538.

Mansour, A.F., Mansour, S.F., \& Abdo, M. (2015). Improvement structural and optical properties of ZnO/PVA nanocomposites. IOSR J. Appl. Phys, 7 (2), 60-69. 
Meyer, T. J. (2009). Photon transport in fluorescent solar collectors. University of Southampton, School of Engineering Sciences. Unpublished PhD Thesis.

Mule, A.V., Villalaz, R., Gaylord, T. K., \& Meird, J.D. (2004). IEEE Photonics Technology letters. 16(11), 2490-2492.

Muna, M., Abbas, M. H., \& Abdallah, J. A. (2014). Synthesis and optical characterization of Nickel Doped Poly Vinyl Alcohol Films. SOP Transactions on Physical Chemistry, 1(2), 1-9.

Mushtak, A., Jabbar, T. J., Alwan, H.L., Mansour, H., \& Hasan, A. (2011). Some Optical Parameters of PMMA Films Doped with $\mathrm{CuSO}_{4}$. The Proceedings of the 18th Scientific Conference for College of Education, Vol. (2), P. 344-348.

Raja, V, Sarma, A.K., \& Narasimha, V. (2003). Optical properties of pure and doped PMMA-COP4VPNO polymer films. Materials Letters, 57, 4678-4683.

Shanshool, H., Yahaya, M., Yunus, W. M., \& Abdullah, L. (2017). Influence of CuO nanoparticles on third order nonlinearity and optical limiting threshold of polymer/ZnO nanocomposites. Opt. Quant. Electron, 49 (18), 1-21.

Susilawati., \& Aris, D. (2009). Dose Response and Optical Properties of Dyed Poly Vinyl AlcoholTrichloroacetic Acid Polymeric Blends Irradiated with Gamma-Rays. American Journal of Applied Sciences, 6, 2071-2077.

Sze, S. (2007). Semiconductor devices physics and technology. 3rd ed. (John Wiley \& Sons, Inc., Canada.

Tauc, J. (1979). Amorphous and liquid semiconductors. New York: Plenum Press, New York.

Zein, K., \& Mohamed, B. (2019). Effect of annealed and Mg-doped nano ZnO on physical properties of PVA. Journal of Molecular Structure. 1181, 507-517 